SVU- International Journal of Veterinary Sciences, 4 (4): 68-84, 2021.

Print ISSN: 2535-1826

\title{
Annotated record of digenean parasites collected from fishes of the River Nile in Qena and Aswan governorates, Egypt
}

\section{Ali Mansour ${ }^{1, *}$, Abdel-Nasser A. Hussein ${ }^{1}$, Khalaf N. Ammar ${ }^{1}$, and Kareem Morsy ${ }^{2,3}$}

${ }^{1}$ Department of Zoology, Faculty of Science, South Valley University, Qena 83523, Egypt, ${ }^{2}$ Department of Zoology, Faculty of Science, Cairo University, Cairo, Egypt,

${ }^{3}$ Biology Department, Faculty of Science, King Khalid University, Abha, Saudi Arabia.

\section{Abstract}

A general investigation of digenean parasites was carried out from four fish species; Fahka puffer fish (Tetraodon lineatus), Nile tilapia (Oreochromis niloticus), Sharp tooth catfish (Clarias gariepinus) and Bayad (Bagrus bajad) from different localities of the River Nile in Qena and Aswan governorates, Egypt during October 2015 to August 2016. Four adult digeneans were found, belonging to four genera; Astiotrema, Orientocreadium, Glossidium and Acanthostomum. These were Astiotrema impletum 90\% (27/30), Orientocreadium batrachoides 83.33\% (25/30), Glossidium pedatum 60\% (12/20) and Acanthostomum spiniceps 86.66\% (26/30). Additionally, two larval trematodes (metacercariae) were detected, belonging to two genera; Clinostomum and Euclinostomum. These were Clinostomum complantum $85.57 \%$ (60/70) and Euclinostomum heterostomum 70\% (35/50). Morphological and morphometric characters were discussed for each species in details and compared with the previously published data. This study considered Glossidium pedatum, Acanthostomum spiniceps and Euclinostomum heterostomum as new records from the River Nile in Qena Governorate.

Keywords: Digenea, Fish, River Nile, Egypt.

DOI: 10.21608/svu.2021.94053.1148 Received: September 3, 2021 Accepted: November 16, 2021 Published: December 25, 2021 *Corresponding Author: Ali Mansour E-mail: alimansour5661@yahoo.com

Citation: Mansour et al., Annotated record of digenean parasites collected from fishes of the River Nile in Qena and Aswan governorates, Egypt. SVU-IJVS 2021, 4 (4): 68-84.

Copyright: (C) Mansour et al. This is an open access article distributed under the terms of the creative common attribution license, which permits unrestricted use, distribution and reproduction in any medium provided the original author and source are created.

Competing interest: The authors have declared that no competing interest exists. 


\section{Introduction}

Fresh water includes $41.24 \%$ of all known fish species. Freshwater fishes are one of the important sources of parasitic infection to human and fish-eating mammals (Mohamed, 1996). Adult trematodes are normally endoparasitic in the gasterointestinal tract, blood vessels or other natural body cavities of vertebrates (Hassan, 2006). Adult trematodes in fishes are usually host specific and rarely cause significant harm to their hosts, although there are some notable exceptions (Paperna and Dzikowski, 2006). The main pathogenic effects from trematode infections in fish hosts occur from larval metacercariae. Metacercariae tend to be less specific host than adult fluke and may affect fish growth and survival, as well as being a source of infection for final hosts and other fish-eating vertebrates, including people (Paperna and Dzikowski, 2006).

In the River Nile, Egypt, digeneans have been studied principally by many authors (Looss, 1896; Looss, 1899; Fischtal and Kuntz, 1963 a,b,c; El-Naffar, 1970; Moravec, 1976; El-Naffar, 1984; ElShahawi and Al-Bassel, 1992; Soliman and El-Damarany, 1995; Hamada, 2002; Saad, 2007; El-Siefy, 2008; El-Mansy et al., 2011; Mansour, 2012; Morsy et al., 2013; Abdel-Gaber, 2015; El-Shahawy et al., 2017). The present study considered Glossidium pedatum, Acanthostomum spiniceps and Euclinostomum heterostomum as first records from the River Nile in Qena Governorate, Egypt.

This study aimed to conduct a general examination of some larval and adult digeneans infecting four species of important freshwater fishes from Qena and Aswan governorates in Egypt. The present study also aimed to improve the existing data on digeneans of freshwater fishes and to provide a preliminary estimation of digenean biodiversity in different localities along the River Nile, Egypt.

\section{Material and Methods}

Fish were caught from commercial fishermen from different localities of the River Nile in Qena and Aswan governorates, Egypt during the period from October 2015 to August 2016. The collected fish were immediately transported to Parasitology Laboratory, Zoology Department, Faculty of Science, South Valley University, Qena, Egypt using special tanks supplied with ice.

Fish samples were dissected and examined for digenean worms. Macroscopic examination of the different organs carried out for the detection of any visible metacercariae. The alimentary canal was opened by a fine pair of scissors and left in an isotonic saline solution $(0.9 \%$ $\mathrm{NaCl}$ ) for few minutes in a petri-dish with occasional shaking (for the parasites to set free from the tissue of the alimentary canal). Repeated changes of the saline solution were done to keep it clear, then the alimentary canal was examined using a binocular dissecting microscope.

The recovered parasites were transferred to a clean saline solution using a brush or small pipettes and washed several times by saline solution to remove any mucous or debris which is usually adhere to their body surface. Worms were placed in between two glass slides or between a glass slide and cover slip in 5\% formalin, subjected to a pressure until relaxed; some worms were preserved in 5\% formalin and others in $70 \%$ ethanol. Worms were stained with acetic acid alum carmine for 5-10 minutes (Carleton, 1967), dehydrated in an ascending series of ethyl alcohol, cleared in xylene, mounted in DPX, covered with cover slip and finally they were examined under light microscope.

\section{Results}

Superfamily: Plagiorchioidea Lühe, 1901

Family: Opisthorchiidae Braun, 1901 Astiotrema impletum (Looss, 1899) Looss, 1900 
Synonym: Astia impleta Looss, 1899

Host: Puffer fish, Tetraodon lineatus

Linnaeus, 1758 (Tetraodontidae)

Infection site: Intestine

Locality: The River Nile in Qena

Governorate (Locality of Dandara), Egypt.

Prevalence: $90 \%$ (27/30).

Specimens deposited in Parasitology Laboratory at Zoology Department, Faculty of Science, South Valley University, Qena, Egypt

\section{Description: (Table 1, Fig. 1A):}

Body was oval in shape and measured $1.63-2.54(1.75 \pm 0.02) \mathrm{mm}$ in length and $0.63-0.98(0.75 \pm 0.02) \mathrm{mm}$ in width. The tegument provided with spines in the anterior third of the body. Oral sucker measured $0.12-0.3 \quad(0.19 \pm 0.02) \mathrm{mm}$ in length by $0.13-0.34(0.25 \pm 0.02) \mathrm{mm}$ in width. Ventral sucker measured 0.11-0.18 $(0.16 \pm 0.02) \mathrm{mm}$ in length and $0.15-0.21$ $(0.18 \pm 0.02)$ in width. Mouth was subterminal and led to a well-developed spherical to oval pharynx which measured
0.09-0.95 $(0.52 \pm 0.02) \mathrm{mm}$ long by $0.06-$ $0.12(0.09 \pm 0.002) \mathrm{mm}$ wide. Pharynx was followed by a long esophagus which bifurcated into two simple intestinal caeca which extended posteriorly to the level of the posterior testis.

Testes were smooth, more or less rounded and more diagonal in position. Anterior testis was $0.22-0.26(0.25 \pm 0.02)$ $\mathrm{mm}$ in length, 0.24-0.41 $(0.32 \pm 0.02) \mathrm{mm}$ in width and posterior testis measured 0.24$0.32(0.29 \pm 0.02) \mathrm{mm}$ long by $0.26-0.5$ $(0.42 \pm 0.02) \mathrm{mm}$ wide. Ovary was spherical, measured $0.13-0.16 \quad(0.15 \pm 0.02) \mathrm{mm}$ in length and $0.12-0.17(0.14 \pm 0.02)$ in width. Vitelline follicles occupied lateral fields extended from the level of intestinal bifurcation to the ends of the intestinal caeca.

Uterus was convoluted, descended from zone and pass between the two testes to the posterior end of the parasite, before ascending to open in the genital atrium. Eggs were numerous and elongated.

Table 1. Comparative measurements $(\mathrm{mm})$ of $A$. impletum in the present study with those described previously.

\begin{tabular}{lcccc}
\hline \multicolumn{1}{c}{ Reference } & $\begin{array}{c}\text { Fischthal and } \\
\text { Kuntz, 1963a }\end{array}$ & $\begin{array}{c}\text { El-Naffar, } \\
\mathbf{1 9 7 0}\end{array}$ & $\begin{array}{c}\text { El-Naffar et } \\
\text { al., 1984 }\end{array}$ & Present study \\
\hline Host & \multicolumn{3}{c}{ Tetraodon fahaka } \\
Locality & Cairo & Assiut & Assiut & Tetraodon lineatus \\
Body length & $1.105-2.530$ & $1.8-2.8$ & $1.33-3.33$ & $1.63-2.54(1.75 \pm 0.02)$ \\
Body width & $0.405-0.750$ & $0.79-1.14$ & $0.617-1.045$ & $0.63-0.98(0.75 \pm 0.02)$ \\
Oral sucker length & $0.185-0.290$ & $0.26-0.32$ & $0.199-0.380$ & $0.12-0.3(0.19 \pm 0.02)$ \\
Oral sucker width & $0.225-0.360$ & $0.30-0.36$ & $0.228-0.427$ & $0.13-0.34(0.25 \pm 0.02)$ \\
Ventral sucker length & $0.125-0.195$ & $0.16-0.19$ & $0.095-0.218$ & $0.11-0.18(0.16 \pm 0.02)$ \\
Ventral sucker width & $0.130-0.215$ & -------- & $0.123-0.237$ & $0.15-0.21(0.18 \pm 0.02)$ \\
Pharynx length & $0.65-0.105$ & $0.10-0.12$ & $0.085-0.142$ & $0.09-0.95(0.52 \pm 0.02)$ \\
Pharynx width & $0.102-0.150$ & $0.088-0.092$ & $0.076-0.152$ & $0.06-0.12(0.09 \pm 0.02)$ \\
Ant. testis length & $0.195-0.340$ & $0.25-0.29$ & $0.209-0.418$ & $0.22-0.26(0.25 \pm 0.02)$ \\
Ant. testis width & $0.135-0.335$ & $0.26-0.31$ & $0.247-0.332$ & $0.24-0.41(0.32 \pm 0.02)$ \\
Post. testis length & $0.215-0.345$ & $0.25-0.29$ & $0.237-0.0 .437$ & $0.24-0.32(0.29 \pm 0.02)$ \\
Post. testis width & $0.160-0.340$ & $0.26-0.31$ & $0.237-0.313$ & $0.26-0.50(0.42 \pm 0.02)$ \\
Ovary length & $0.120-0.188$ & $0.132-0.188$ & $0.085-0.19$ & $0.13-0.16(0.15 \pm 0.02)$ \\
Ovary width & $0.130-0.185$ & --------- & $0.085-0.196$ & $0.12-0.17(0.14 \pm 0.02)$ \\
\hline
\end{tabular}


Superfamily: Plagiorchioidea Lühe, 1901

Family: Orientocreadiidae Yamaguti, 1958

Orientocreadium batrachoides Tubangui, 1931

Synonym: Ganada barabankiae (Dayal, 1938) Fischthal \& Kuntz, 1963

Host: Sharptooth catfis, Clarias gariepinus Burchell, 1822 (Clariidae).

Infection site: Intestine

Locality: The River Nile in Qena

Governorate (Locality of Dandara), Egypt.

Prevalence: $83.33 \%$ (25/30).

Specimens deposited in Parasitology Laboratory at Zoology Department, Faculty of Science, South Valley University, Qena, Egypt

Description: (Table 2, Fig. 1B):

Body was elongated and measured $1.63-2.82(0.20 \pm 0.2) \mathrm{mm}$ long by $0.21-0.53$ $(0.42 \pm 0.02) \mathrm{mm}$ wide. Cuticle was armed with minute spines. Oral sucker was subterminal and measured 0.14-0.28 $(0.21 \pm 0.02) \mathrm{mm}$ long by $0.12-0.23$ $(0.17 \pm 0.02) \mathrm{mm}$ wide; pharynx measured $0.06-0.17(0.12 \pm 0.02) \mathrm{mm}$ long by 0.12 $0.19(0.17 \pm 0.02) \mathrm{mm}$ wide; intestinal caeca extended behind uterine coils, almost into posterior body margin. Ventral sucker was $0.17-0.31(0.25 \pm 0.02) \mathrm{mm}$ in length and $0.15-0.28(0.19 \pm 0.02) \mathrm{mm}$ in width, it occupied about one third of body length.

Testes were rounded, tandem with anterior testis situated in mid region of body. Anterior testis measured 0.18-0.27 $(0.25 \pm 0.02) \mathrm{mm}$ in length and posterior testis was $0.24-0.39(0.35 \pm 0.02) \mathrm{mm}$ in length. Cirrus sac was also variable depending on the state of contraction, it may curve round to right or left of ventral sucker or may be anterior to it. Common genital pore was median, situated between ventral sucker and bifurcation of intestine. Ovary was rounded, its length was 0.17 $0.28(0.23 \pm 0.02) \mathrm{mm}$ and it often separated from the ventral sucker by cirrus sac and/or seminal vesicle. Receptaculum seminis was not observed.

Uterus with descending and ascending limbs formed a series of irregular transverse coils. Uterine coils separated from the posterior margin of the body by vitelline follicles. Vitellaria lie in two extracaecal bands extended from the level of ventral sucker to posterior extremity. Eggs were numerous and operculated.

Superfamily: Plagiorchioidea Lühe, 1901 Glossidium pedatum Looss, 1899

Host: Sharptooth catfish, Clarias gariepinus Burchell, 1822 (Clariidae).

Infection site: Intestine

Locality: The River Nile in Qena

Governorate (Locality of Al- Ashraf Al-

Qibliyyah), Egypt.

Prevalence: $60 \%$ (12/20).

Specimens deposited in Parasitology Laboratory at Zoology Department, Faculty of Science, South Valley University, Qena, Egypt

Description: (Table 3, Fig. 1C):

Body was entirely spined, slightly rounded anteriorly, bifid and expanded laterally at posterior extremity, measured $1.11-2.36(1.52 \pm 0.2) \mathrm{mm}$ long by $0.21-0.45$ $(0.32 \pm 0.02) \mathrm{mm}$ wide. Oral sucker was slightly rounded to truncate or slightly concave anteriorly, wider than long, measured $0.08-0.28(0.15 \pm 0.02) \mathrm{mm}$ long by $0.18-0.26 \quad(0.19 \pm 0.02) \mathrm{mm}$ wide; acetabulumb was rounded to slightly longitudinally or transversely elongated, measured $0.31-0.42(0.38 \pm 0.02) \mathrm{mm}$ long by $0.17-0.32 \quad(0.26 \pm 0.02)$ wide. Pharynx was $0.04-0.15(0.14 \pm 0.02) \mathrm{mm}$ in length, $0.08-0.12(0.1 \pm 0.02) \mathrm{mm}$ in width, fourlobed anteriorly with dorsal lobe longer and ventral lobe shorter than two lateral ones; esophagus was distinct, thick-walled and muscular.

Testes were longitudinally elongated, smooth, diagonal or sometimes nearly tandem; anterior testis was dextral, measured $0.13-0.35(0.25 \pm 0.02) \mathrm{mm}$ long by $0.14-0.26 \quad(0.46 \pm 0.02) \mathrm{mm}$ wide, posterior testis was sinistral, measured $0.11-0.3(0.15 \pm 0.02) \mathrm{mm}$ long by $0.13-0.26$ $(0.19 \pm 0.02) \mathrm{mm}$ wide. Cirrus sac was thickwalled and muscular. Ovary was sinistral, smooth, longitudinally elongated but 
sometimes nearly rounded, measured 0.11$0.18(0.16 \pm 0.02) \mathrm{mm}$ long by $0.12-0.25$ $(0.23 \pm 0.02) \mathrm{mm}$ wide. Vitelline follicles were overlapping ceca in lateral fields; uterus in mature adults was filling most of hindbody and posttesticular space, lying ventral to gonads and proximal part of cirrus sac, occasionally overlapping ceca and vitellaria laterally. Eggs were yellow to yellow-brown and operculated.

Table 2. Comparative measurements $(\mathrm{mm})$ of $O$. batrachoides in the present study with those described previously.

\begin{tabular}{|c|c|c|c|c|}
\hline References & $\begin{array}{c}\text { Beverley- } \\
\text { Burton, } 1962\end{array}$ & $\begin{array}{l}\text { Abdel-Gaber } \\
\text { et al., 2015 }\end{array}$ & $\begin{array}{l}\text { Dumbo et } \\
\text { al., } 2019\end{array}$ & Present study \\
\hline Host & $\begin{array}{c}\text { Clarias } \\
\text { mossambicus }\end{array}$ & Clarias gariepinus & Clarias gariepinu & us Clarias gariepinus \\
\hline Locality & Rhodesia & Egypt & Moz & Egypt \\
\hline Body length & $1.11-2.58$ & $1.83-2.72$ & $1.47-2.67$ & $1.63-2.82(0.20 \pm 0.2)$ \\
\hline Body width & $0.22-0.77$ & $0.31-0.46$ & $314-663$ & $0.21-0.53$ \\
\hline Oral sucker length & $0.11-0.23$ & $0.16-$ & $0.1-0.2$ & $0.14-0.2$ \\
\hline Oral sucker width & $0.11-0.33$ & $0.15-0.20$ & $0.11-0.22$ & $0.12-0.23(0.17 \pm 0.02)$ \\
\hline Ventral sucker length & $0.12-0.24$ & $0.18-0.23$ & $0.14-0.21$ & $0.17-0.31(0.25 \pm 0.02)$ \\
\hline Ventral sucker width & $0.12-0.25$ & $0.19-0.27$ & $0.11-0.22$ & $0.15-0.28(0.19 \pm 0.02)$ \\
\hline Pharynx length & $0.08-0.16$ & $0.07-0.12$ & $0.11-0.16$ & $0.06-0.17(0.12 \pm 0.02)$ \\
\hline Pharynx width & $0.09-0.16$ & $0.10-0.18$ & $0.08-0.13$ & $0.12-0.19(0.17 \pm 0.02)$ \\
\hline Anterior testis length & $0.11-0.25$ & $0.19-0.24$ & $0.11-0.22$ & $0.18-0.27(0.25 \pm 0.02)$ \\
\hline Posterior testis length & $0.11-0.30$ & $0.21-0.31$ & $0.16-0.3$ & $0.24-0.39(0.35 \pm 0.02)$ \\
\hline Ovary length & $0.13-0.27$ & $0.16-0.21$ & $0.12-0.23$ & $0.17-0.28(0.23 \pm 0.02)$ \\
\hline
\end{tabular}

Table 3. Comparative measurements $(\mathrm{mm})$ of $G$. pedatum in the present study with those described previously.

\begin{tabular}{|c|c|c|c|c|}
\hline References & Fischthal, 1973 & $\begin{array}{c}\text { Mashego, } \\
1977\end{array}$ & $\begin{array}{c}\text { van } \\
\text { Rensburg, } \\
2013 \\
\end{array}$ & Present study \\
\hline Host & Clarias mossambicus & \multicolumn{3}{|c|}{ Clarias gariepinus } \\
\hline Location & Ethiopia & South Africa & Botswana & Egypt \\
\hline Body length & $1.17-1.92$ & 1.93 & $1.25-2.98$ & $1.11-2.36(1.52 \pm 0.2)$ \\
\hline Body Width & $0.2-0.31$ & 0.31 & $0.27-0.55$ & $0.21-0.45(0.32 \pm 0.02)$ \\
\hline Oral sucker length & $0.11-0.15$ & 0.21 & $0.10-0.30$ & $0.08-0.28(0.15 \pm 0.02)$ \\
\hline Oral sucker Width & $0.12-0.17$ & 0.16 & $0.12-0.20$ & $0.18-0.26(0.19 \pm 0.02)$ \\
\hline Acetabulum length & & & & $0.31-0.42(0.38 \pm 0.02)$ \\
\hline Acetabulum width & $0.11-0.15$ & 0.17 & $0.13-0.23$ & $0.17-0.32(0.26 \pm 0.02)$ \\
\hline Pharynx length & 0.06-0.09 & & $0.05-0.10$ & $0.04-0.15(0.14 \pm 0.02)$ \\
\hline Pharynx width & $0.06-0.1$ & & $0.11-0.15$ & $0.08-0.12(0.1 \pm 0.02)$ \\
\hline Ant. testis length & $0.1-0.13$ & 0.15 & $0.12-0.24$ & $0.13-0.35(0.25 \pm 0.02)$ \\
\hline Ant. testis width & $0.08-0.1$ & 0.11 & $0.16-0.24$ & $0.14-0.26(0.46 \pm 0.02)$ \\
\hline Post. testis length & $0.12-0.17$ & 0.2 & $0.12-0.24$ & $0.11-0.3(0.15 \pm 0.02)$ \\
\hline Post. testis width & $0.08-0.11$ & 0.11 & $0.13-0.24$ & $0.13-0.26(0.19 \pm 0.02)$ \\
\hline Ovary length & $0.07-0.11$ & 0.12 & $0.10-0.19$ & $0.11-0.18(0.16 \pm 0.02)$ \\
\hline Ovary width & 0.06-0.09 & 0.11 & $0.10-0.20$ & $0.12-0.25(0.23 \pm 0.02)$ \\
\hline
\end{tabular}


Superfamily: Opisthorchioidea Looss, 1899

Family: Cryptogonimidae Ward, 1917

Acanthostomum spiniceps (Looss, 1896) Looss, 1899

Synonym: Distomum spiniceps Looss, 1896

Host: Bayad, Bagrus bajad Forskål, 1775

(Bgridae).

Infection site: Intestine

Locality: The River Nile in Qena

Governorate (Locality of Dandara), Egypt.

Prevalence: $86.66 \%$ (26/30).

Specimens deposited in Parasitology Laboratory at Zoology Department, Faculty of Science, South Valley University, Qena, Egypt

Description: (Table 4, Fig. 1D):

Body length was 1.21-1.63 (1.45 \pm 0.2$)$ $\mathrm{mm}$ in length and 0.3-0.52 $(0.41 \pm 0.02) \mathrm{mm}$ in width. Body surface was covered with fine spines, which were most numerous on the anterior portion of body and became more scarce toward the posterior end. Oral sucker was funnel-shaped, measured 0.15$0.4(0.35 \pm 0.02) \mathrm{mm}$ in length and 0.23-0.47 $(0.30 \pm 0.02) \mathrm{mm}$ in width. Outer surface of this sucker was armed with a row of large simple spines. Ventral sucker was almost rounded, measured 0.12-0.28 (0.25 \pm 0.02$)$ $\mathrm{mm} \times 0.1-0.29(0.24 \pm 0.02) \mathrm{mm}$. Pharynx was strongly muscular and oval, measured 0.04-0.15 (0.07 \pm 0.02$) \quad \mathrm{mm} \quad \mathrm{x} \quad 0.05-0.11$ $(0.08 \pm 0.02) \mathrm{mm}$. Esophagus was relatively short. Intestinal caeca were relatively narrow and run along the body approximately up to the level of the posterior margin of the second testis, where they open to the body surface by distinct anal openings.

Testes were tandem and located near the posterior end of the body. Anterior testis measured $0.12-0.33(0.23 \pm 0.02) \mathrm{mm}$ long $x$ 0.23-0.41 $(0.34 \pm 0.02) \mathrm{mm}$ wide and posterior testis measured 0.11-0.34 $(0.22 \pm 0.02) \quad \mathrm{mm}$ long $\mathrm{x} \quad 0.2-0.26$ $(0.23 \pm 0.02) \mathrm{mm}$ wide. Ovary was almost spherical and subterminal, measured 0.15 $0.2 \quad(0.17 \pm 0.02) \quad$ long and 0.12-0.19 $(0.15 \pm 0.02)$ wide. Vitellaria were follicular and situated on sides of body in its posterior half.

Family: Clinostomidae Lühe, 1901

Subfamily: Clinostominae Lühe, 1901

Clinostomum complantum (Rudolphi, 1814) Braum, 1899

\section{Synonym: Clinostomum \\ marginatum (Rudolphi, 1819) Braun, 1899}

Host: Nile tilapia, Oreochromis niloticus

Linnaeus, 1758 (Cichlidae)

Infection site: Buccal cavity and heart

Locality: The River Nile in Aswan

Governorate (Locality of Shniarha), Egypt.

Prevalence: $85.57 \%$ (60/ 70).

Specimens deposited in Parasitology Laboratory at Zoology Department, Faculty of Science, South Valley University, Qena, Egypt

Description: (Table 5, Fig. 1E):

Body was linguiform and aspinose with blunt ends, measured 2.12-5.17 (3.89 \pm 0.2$) \mathrm{mm}$ in length and 1.74-2.36 $(2.15 \pm 0.2) \mathrm{mm}$ in width. Oral sucker was sub-terminal and measured 0.13-0.21 $(0.17 \pm 0.02) \mathrm{mm}$ long by 0.14 $0.35(0.25 \pm 0.02) \mathrm{mm}$ wide. Ventral sucker situated at the anterior third of the body, measured $0.42-0.86(0.65 \pm 0.02) \mathrm{mm}$ long and $0.4-0.88(0.66 \pm 0.02) \mathrm{mm}$ wide. Mouth occupied the center of oral sucker and opened into a small prepharynx and the pharynx which measured 0.1-0.21 $(0.15 \pm 0.02) \mathrm{mm} \times 0.03-0.09(0.05 \pm 0.02)$ $\mathrm{mm}$.

Caeca occupied a greater part of the body on either side and supported with small lateral diverticula. Testes were large digitate; anterior testis was $0.15-0.28$ $(0.22 \pm 0.02) \mathrm{mm} \times 0.14-0.49(0.3 \pm 0.02) \mathrm{mm}$ and posterior testis was $0.14-0.36$ $(0.26 \pm 0.02) \mathrm{mm} \times 0.11-0.52(0.33 \pm 0.02)$ $\mathrm{mm}$. Ovary was small, rounded to oval and intertesticular, measured 0.08-0.27 $(0.17 \pm 0.02) \mathrm{mm} \times 0.06-0.24(0.16 \pm 0.02)$ $\mathrm{mm}$. Excretory bladder was small, Vshaped and opened out by a sub-terminal pore. 
Mansour et al., 2021

SVU-IJVS, 4 (4): 68-84

Table 4. Comparative measurements $(\mathrm{mm})$ of $A$. spiniceps in the present study with those described previously.

\begin{tabular}{|c|c|c|c|c|}
\hline References & $\begin{array}{l}\text { Fernandes } \\
\text { et al., 2002 }\end{array}$ & $\begin{array}{l}\text { Morsy et al., } \\
2013\end{array}$ & Abdel-Gaber et al., 2018 & Present study \\
\hline Host & $\begin{array}{l}\text { Astroscopus } \\
\text { sexspinosus }\end{array}$ & $\begin{array}{c}\text { Lates } \\
\text { niloticus }\end{array}$ & Lates niloticus & Bagrus bajad \\
\hline Locality & Brazil & Egypt & Egypt & Egypt \\
\hline Body length & 3.37 & $1.203-2.074$ & $1.291-2.871(2.210 \pm 0.1)$ & $1.2-1.63(1.45 \pm 0.2)$ \\
\hline Body width & 0.57 & $0.311-0.534$ & $0.298-0.476(0.392 \pm 0.01)$ & $0.30-0.52(0.41 \pm 0.02)$ \\
\hline $\begin{array}{l}\text { Oral sucker } \\
\text { length }\end{array}$ & 0.318 & $0.201-0.353$ & $0.232-0.397(0.321 \pm 0.01)$ & $0.15-0.40(0.35 \pm 0.02)$ \\
\hline $\begin{array}{l}\text { Oral sucker } \\
\text { width }\end{array}$ & 0.270 & $0.245-0.310$ & $0.257-0.344(0.322 \pm 0.01)$ & $0.23-0.47(0.30 \pm 0.02)$ \\
\hline $\begin{array}{l}\text { Ventral } \\
\text { sucker length }\end{array}$ & 0.150 & $0.110-0.140$ & $0.123-0.151(0.143 \pm 0.01)$ & $0.12-0.28(0.25 \pm 0.02)$ \\
\hline $\begin{array}{l}\text { Ventral } \\
\text { sucker width }\end{array}$ & 0.138 & $0.120-0.204$ & $0.132-0.198(0.157 \pm 0.01)$ & $0.10-0.29(0.24 \pm 0.02)$ \\
\hline Pharynx & $\begin{array}{c}0.138 \times \\
0.135\end{array}$ & $\begin{array}{c}0.163-0.177 \\
\times 0.150- \\
0.190\end{array}$ & $\begin{array}{c}0.154-0.173(0.165 \pm 0.01) \\
\times 0.162-0.189 \\
(0.175 \pm 0.01)\end{array}$ & $\begin{array}{c}0.04-0.15 \times 0.05-0.11 \\
(0.07 \pm 0.02 \times 0.08 \pm 0.02)\end{array}$ \\
\hline anterior testis & 0.285 & $\begin{array}{c}0.130- \\
0.220 \times 0.314- \\
0.358\end{array}$ & $\begin{array}{c}0.128-0.286(0.212 \pm 0.01) \\
\times 0.343-0.430 \\
(0.358 \pm 0.01)\end{array}$ & $\begin{array}{c}0.12-0.33 \times 0.23-0.41 \\
(0.23 \pm 0.02 \times 0.34 \pm 0.02)\end{array}$ \\
\hline posterior testis & $0.315 \times 0.281$ & $\begin{array}{c}0.180- \\
0.274 \times 0.390- \\
0.327\end{array}$ & $\begin{array}{c}0.194-0.287(0.254 \pm \\
0.01) \times 0.354-0.420 \\
(0.386 \pm 0.01)\end{array}$ & $\begin{array}{c}0.11-0.34 \times 0.2-0.26 \\
(0.22 \pm 0.02 \times 0.23 \pm 0.02)\end{array}$ \\
\hline Ovary length & 0.243 & $0.102-0.166$ & $0.143-0.178(0.156 \pm 0.01)$ & $0.15-0.2(0.17 \pm 0.02)$ \\
\hline Ovary width & 0.187 & $0.100-0.164$ & $0.098-0.143(0.121 \pm 0.01)$ & $0.12-0.19(0.15 \pm 0.02)$ \\
\hline No. spines & 27 & 23 & 23 & 23 \\
\hline
\end{tabular}

Table 5. Comparative measurements $(\mathrm{mm})$ of $C$. complantum in the present study with those described previously.

\begin{tabular}{|c|c|c|c|}
\hline Reference & Malik et al., 2016 & Simsek et al., 2018 & Present study \\
\hline Host & ---------------- & ---------------- & Oreochromus niloticus \\
\hline Location & ---------------- & ----------------- & Egypt \\
\hline Body length & $1.917-2.700(0.231 \pm 0.2)$ & $3.998-6.718(5.1 \pm 0.34)$ & $2.12-5.17(3.89 \pm 0.2)$ \\
\hline Body width & $0.64-1.05(0.08 \pm 0.1)$ & $1.197-2.131(1.69 \pm 0.16)$ & $1.74-2.36(2.15 \pm 0.2)$ \\
\hline Oral sucker length & $0.14-0.23(0.17 \pm 0.02)$ & $0.24-0.31(0.27 \pm 0.01)$ & $0.13-0.21(0.17 \pm 0.02)$ \\
\hline Oral sucker width & $0.14-0.24(0.19 \pm 0.02)$ & $0.26-0.48(0.33 \pm 0.03)$ & $0.14-0.35(0.25 \pm 0.02)$ \\
\hline $\begin{array}{l}\text { Ventral sucker } \\
\text { length }\end{array}$ & $0.33-0.44(0.39 \pm 0.03)$ & $0.58-0.82(0.68 \pm 0.03)$ & $0.42-0.86(0.65 \pm 0.02)$ \\
\hline $\begin{array}{l}\text { Ventral sucker } \\
\text { width }\end{array}$ & $0.32-0.42(0.37 \pm 0.02)$ & $0.67-0.89(0.76 \pm 0.03)$ & $0.4-0.88(0.66 \pm 0.02)$ \\
\hline Pharynx & 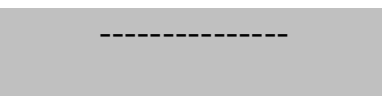 & 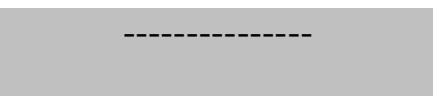 & $\begin{array}{c}0.10-0.21 \times 0.03-0.09 \\
(0.15 \pm 0.02 \times 0.05 \pm 02)\end{array}$ \\
\hline Anterior testis & $\begin{array}{c}0.11-0.20(0.15 \pm 0.02) \times \\
0.08-0.26(0.14 \pm 0.02)\end{array}$ & $\begin{array}{c}0.29-0.71(0.47 \pm 0.06) \times \\
0.2-0.49(0.4 \pm 0.04)\end{array}$ & $\begin{array}{c}0.15-0.28 \times 0.14-0.49 \\
(0.22 \pm 0.02 \times 0.3 \pm 02)\end{array}$ \\
\hline Posterior testis & $\begin{array}{c}0.1-0.19(0.14 \pm 0.028) \times \\
0.1-0.32(0.21 \pm 0.05)\end{array}$ & $\begin{array}{c}0.21-0.39(0.32 \pm 0.02) \times \\
0.38-0.56(0.45 \pm 0.02)\end{array}$ & $\begin{array}{c}0.14-0.36 \times 0.11-0.52 \\
(0.26 \pm 0.02 \times 0.33 \pm 02)\end{array}$ \\
\hline Ovary & $\begin{array}{c}0.03-0.1(0.06 \pm 0.02) \times \\
0.04-0.08(0.05 \pm 0.02)\end{array}$ & $\begin{array}{c}0.12-0.15(0.13 \pm 0.005) \times \mathrm{x} \\
0.08-0.14(0.11 \pm 0.009)\end{array}$ & $\begin{array}{c}0.08-0.27 \times 0.06-0.24 \\
(0.17 \pm 0.02 \times 0.16 \pm 02)\end{array}$ \\
\hline
\end{tabular}


Family: Clinostomidae Lühe, 1901

Subfamily: Euclinostominae Yamaguti, 1958

Euclinostomum heterostomum (Rudolphi, 1809) Travassos, 1928

Host: Nile tilapia, Oreochromis niloticus Linnaeus, 1758 (Cichlidae).

Infection site: Buccal cavity and kidney

Locality: The River Nile in Qena

Governorate (Locality of Al- Ashraf Al-

Qibliyyah), Egypt.

Prevalence: $70 \%$ (35/ 50).

Specimens deposited in Parasitology Laboratory at Zoology Department, Faculty of Science, South Valley University.

Description: (Table 6, Fig. 1F):

Body was stout, fleshy, linguiform, attenuated anteriorly and slightly rounded posteriorly, measured 4.11-5.52 (4.55 \pm 0.2$)$ $\mathrm{mm}$ long and 1.53-2.67 (1.72 \pm 0.02$) \mathrm{mm}$ wide. Cuticle was thin and smooth. Oral sucker was small and subterminal, measured $0.16-0.34(0.26 \pm 0.02) \mathrm{mm}$ long and 0.2-0.76 $(0.52 \pm 0.02) \mathrm{mm}$ wide. Ventral sucker was well developed, strongly muscular, almost rounded marginally and heart shaped internally, situated at the end of the first quarter of the body, measured $0.52-1.04(0.83 \pm 0.02) \mathrm{mm}$ long and 0.72$0.96(0.85 \pm 0.02) \mathrm{mm}$ wide. Pharynx was short and measured 0.05-0.08 $(0.06 \pm 0.002)$ $\mathrm{mm} \quad \mathrm{x} \quad 0.04-0.07 \quad(0.07 \pm 002) \quad \mathrm{mm}$. Esophagus was very short and bifurcated into long diverticulated ceca extended up to posterior extremity. Left cecum was supported with seven simple branches while right cecum with 12 simple branches.

Caecal branches were elongated, lobed and appeared finger like processes with somewhat swollen extremities. Testes were asymmetrical, tandem and situated in third quarter of body. Anterior testis was wider than long and measured 0.01-0.02 $(0.01 \pm 0.002) \mathrm{mm}$ long $\mathrm{x}$ 0.51-0.61 $(0.06 \pm 002) \mathrm{mm}$ wide. Posterior testis was also asymmetrical, slightly lobed and measured 0.16-0.22 (0.19 \pm 0.02$) \mathrm{mm}$ long $\mathrm{x}$ 0.59-0.63 (0.61 \pm 002$) \mathrm{mm}$ wide. Ovary was almost rounded, small, lie laterally on upper corner of anterior testis and measured 0.13$0.14 \quad(0.13 \pm 0.02) \quad \mathrm{mm} \quad \mathrm{x} \quad 0.12-0.15$ $(0.14 \pm 002) \mathrm{mm}$.

Table 6. Comparative measurements $(\mathrm{mm})$ of $E$. heterostomum in the present study with those described previously.

\begin{tabular}{|c|c|c|c|c|}
\hline Reference & Rai, 1970 & $\begin{array}{c}\text { Jhansilakshmibai } \\
\text { and Madhavi, } 1997\end{array}$ & $\begin{array}{l}\text { Vankara, } \\
2011 \\
\end{array}$ & Present study \\
\hline Host & & Channa punctatus & & Oreochromis niloticus \\
\hline Location & India & India & India & Egypt \\
\hline Body length & $5.41-5.62$ & $0.183-4.534$ & $3.45-8.41$ & $4.11-5.52(4.55 \pm 0.2)$ \\
\hline Body width & $1.46-2.32$ & $0.089-1.627$ & $1.28-2.36$ & $1.53-2.67(1.72 \pm 0.02)$ \\
\hline Oral sucker length & $0.18-0.23$ & $0.042-0.168$ & $0.14-0.34$ & $0.16-0.34(0.26 \pm 0.02)$ \\
\hline Oral sucker width & $0.21-0.25$ & $0.05-0.217$ & $0.20-0.52$ & $0.2-0.76(0.52 \pm 0.02)$ \\
\hline $\begin{array}{l}\text { Ventral sucker } \\
\text { length }\end{array}$ & 0.75 & $0.07-0.929$ & $0.60-1.16$ & $0.52-1.04(0.8$ \\
\hline $\begin{array}{l}\text { Ventral sucker } \\
\text { width }\end{array}$ & $0.82-0.87$ & $0.07-0.914$ & $0.55-1.34$ & $0.72-0.96(0.85 \pm 0.02)$ \\
\hline Pharynx & $\begin{array}{c}0.06-0.09 x \\
0.05-0.08\end{array}$ & & $\begin{array}{l}0.137-0.17 \\
\times 0.16-0.44\end{array}$ & $\begin{array}{l}0.053-0.089 \times 0.041-0.076 \\
(0.063 \pm 0.002 \times 0.072 \pm 002)\end{array}$ \\
\hline Anterior testis & $\begin{array}{c}0.013-0.014 \mathrm{x} \\
0.61-0.72\end{array}$ & $\begin{array}{c}0.126-0.314 \times 0.150- \\
0.491\end{array}$ & $\begin{array}{l}0.22-0.63 \mathrm{x} \\
0.31-0.78\end{array}$ & $\begin{array}{c}0.010-0.023 \times 0.51-0.61 \\
(0.013 \pm 0.002 \times 0.060 \pm 002)\end{array}$ \\
\hline Posterior testis & $\begin{array}{c}0.18-0.21 x \\
0.51-0.52\end{array}$ & $\begin{array}{c}0.111-0.370 \times 0.145- \\
0.420\end{array}$ & $\begin{array}{c}0.07-0.39 \mathrm{x} \\
0.10-0.22\end{array}$ & $\begin{array}{c}0.16-0.22 \times 0.59-0.42 \\
(0.19 \pm 0.02 \times 0.61 \pm 002)\end{array}$ \\
\hline Ovary & $\begin{array}{c}0.12-0.15 \mathrm{x} \\
0.12-0.14\end{array}$ & $\begin{array}{c}0.052-0.136 \times 0.050- \\
0.146\end{array}$ & ------------- & $\begin{array}{c}0.13-0.14 \times 0.12-0.15 \\
(0.13 \pm 0.02 \times 0.14 \pm 0.02)\end{array}$ \\
\hline
\end{tabular}




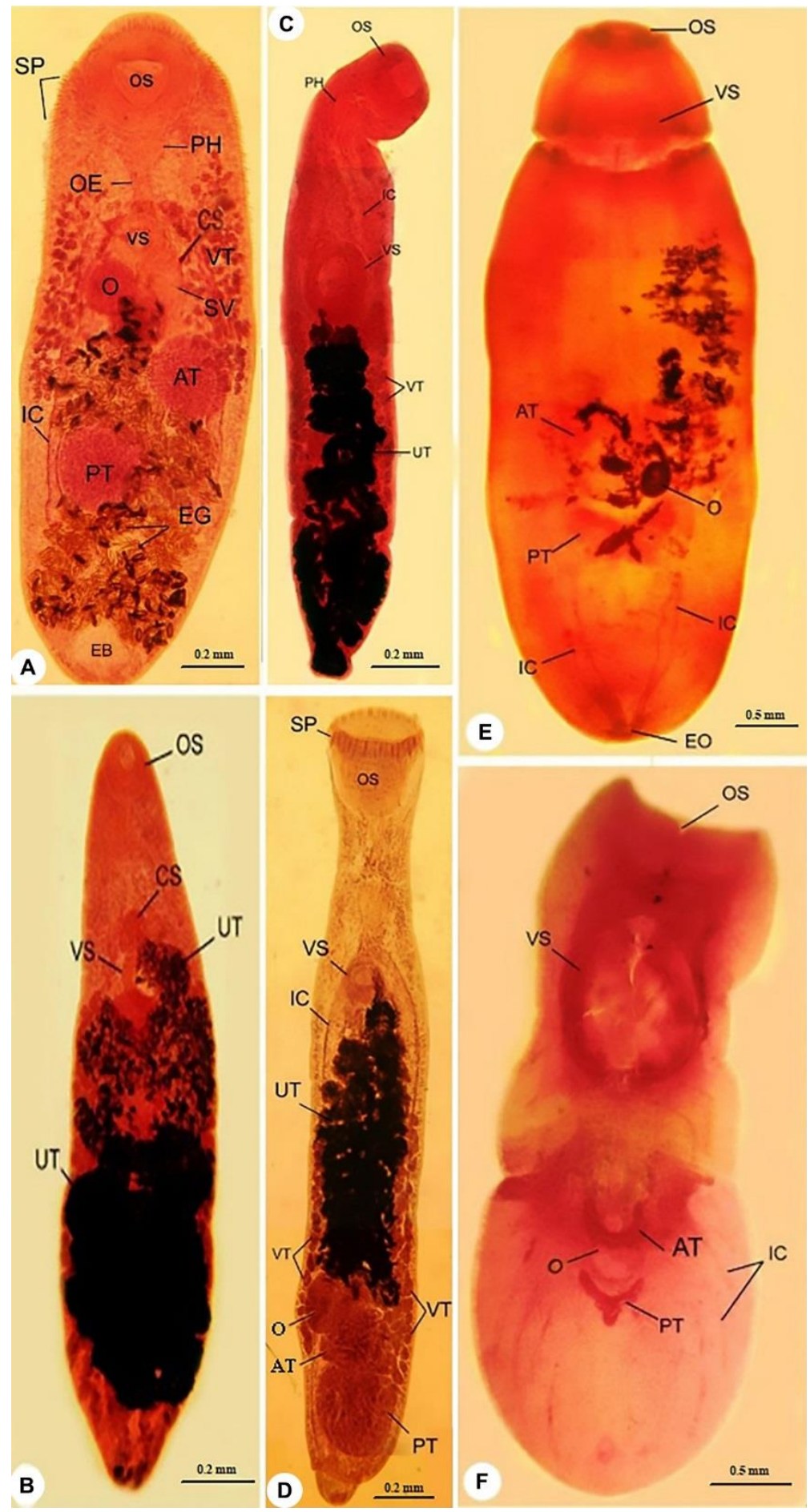

Fig. 1. Light photomicrographs of the recorded digenean parasites among the examined fish species from Qena and Aswan governorates, Egypt. (1A): Whole mount preparation of the adult worm Astiotrema impletum with an oval body, consists of: a tegument provided with spines (SP) in the anterior third of the body, an oral sucker (OS), followed by pharynx $(\mathrm{PH})$, oesophagus $(\mathrm{OE})$, small median ventral sucker (VS), two intestinal caeca (IC), spherical ovary $(\mathrm{O})$, convoluted uterus filled with numerous and elongated eggs (EG), two testes, anterior testis (AT) and posterior testis (PT), seminal vesicle (SV), cirrus sac (CS), laterally distributed vitellaria (VT) and an excretory bladder (EB), scale bar $0.2 \mathrm{~mm}$. (1B): Whole mount preparation of the adult worm Orientocreadium batrachoides with elongated body consists of: an oral sucker (OS), ventral sucker (VS), uterus (UT) filled with numerous and small eggs and cirrus sac (CS), scale bar 0. $2 \mathrm{~mm}$. (1C): Whole mount preparation of the adult worm Glossidium pedatum with an elongated oral sucker (OS) leading to pharynx $(\mathrm{PH})$, intestinal caeca (IC), ventral sucker (VS), uterus (UT) and lateral vitellaria (VT), scale bar $0.2 \mathrm{~mm}$. (1D): Whole mount preparation of the adult worm Acanthostomum spiniceps with elongated body consists of: a funnel-shaped oral sucker (OS) armed with a row of large simple spines (SP), ventral sucker (VS), intestinal caeca (IC), small oval ovary (O), uterus (UT) filled with numerous and mature eggs, anterior testis (AT), posterior testis (PS) and laterally scattered vitellaria (VT), scale bar $0.2 \mathrm{~mm}$. (1E): Whole mount preparation of digenean metacercaria Clinostomum complantum showing a subterminal oral sucker (OS), ventral sucker (VS), intestinal caeca (IC), small rounded to oval ovary $(\mathrm{O})$, anterior testis (AT), posterior testis (PT) and an excretory opening (EO), scale bar $0.5 \mathrm{~mm}$. (1F): Whole mount preparation of digenean metacercaria Euclinostomum heterostomum consisting of a small oral sucker (OS), well developed ventral sucker (VS), diverticulated intestinal caeca (IC), a small ovary (O), tandem testis; anterior testis (AT) and posterior testis (PT), scale bar $0.5 \mathrm{~mm}$. 


\section{Discussion}

Astiotrema impletum (Looss, 1899) Looss, 1900:

Astiotrema impletum was originally described by Looss (1899) from Tetraodon lineatus (as Tetradon fahaka) (Tetraodontidae) in the Nile at Cairo, Egypt. Looss (1900) proposed the name Astiotrema and both species Distoma reniferum and Astia impleta were renamed to A. renferuim (Looss, 1898) Looss, 1900 and A. impletum (Looss, 1898) Looss, 1900 respectively. This species is known only from North Africa from the freshwater fish, Tetraodon lineatus (as Tetraodon fahaka). Further studies reported Astiotrema impletum (Looss, 1899) Loss, 1900 from different localities of Egypt among Tetraodon lineatus (as Tetradon fahaka) (Fischthal and Kuntz, 1963a; El-Naffar, 1970; El-Naffar, 1984; El-Siefy, 2008), Tetraodon lineatus (Karar et al., 2021) and Oreochromis niloticus (Hamada, 2002) and from Sudan among Tetraodon lineatus (as Tetradon fahaka) (Odhner, 1911; Yeh and Fotedar, 1958; Khalil, 1969).

This species is distinguished from the rest members of Astiotrema by the following characters; the oral sucker is large and twice that of the ventral, the caeca is short in which it terminates at the posterior border of the second testis or the junction of the third- and fourth-quarters of the body and vitellaria are restricted to anterior half of the worm. The eggs in our specimens were elongated, thin shell operculated whereas no mention was made of this by Looss (1899), Odhner (1911) and Yeh and Fotedar (1958).

Odhner (1911) noted that the testes occasionally were slightly lobed and almost symmetrical; whereas, all the previous authors and the present study noted smooth testes at all times and a more diagonal position. Testes in the present study were separated by a distinct intertesticular space while Odhner (1911) and Fischthal \& Kuntz (1963a) noted that the testes were in contact or closer together. El-Naffar (1984) stated that vitellaria in his specimens extending from the level of intestinal bifurcation to the end of the intestinal caeca but his illustration shows that the distribution of vitellaria reaches only to the beginning of posterior testis. Morphometry of A. impletum specimens collected by the previous authors were comparatively similar to our specimens as shown in Table 1.

\section{Orientocreadium batrachoides Tubangui, 1931:}

Orientocreadium batrachoides was first described by Tubangui (1931) from Clarias batrachus in Philippines. More studies were carried out on this species (Beverley-Burton, 1962; Yamaguti, 1971; Tang and Lin, 1973; Sirikantayakul, 1976; Moravec, 1977; Moravec and Sey, 1989; Ruangpan, 1989; Tepe et al., 2013; Bhure and Nanware, 2011; Bhure et al., 2013; Bhure and Nanware, 2016; Zhokhov et al., 2017; Dumbo et al., 2019). This species was previously recorded from Egypt in Clarias gariepinus (Abdel-Gaber et al., 2015; El-Siefy et al., 2017; Attia et al., 2021) or Clarias gariepinus (as Clarias lazera) (Fischthal and Kuntz, 1963b; ElNaffar et al., 1983; Negm El-Din et al., 1988; El-Naggar et al., 1993a,b; El-Seify et al., 1997; El-Mansy et al., 2011; ElShahawy et al., 2017).

The presence of unipartite external seminal vesicles and vitelline follicles that start at the level of the ovary until they meet at the posterior end of the body distinguishes the species. The vitelline follicles are grouped in two lateral fields limited in the middle of the body, according to Bhure and Nanware (2011) and AbdelGaber et al. (2015). In our specimens, external seminal vesicle was not observed. Measurements of the present specimens were very compatible with those previously described, as indicated in Table 2.

\section{Glossidium pedatum Looss, 1899:}

Looss (1899) reported Glossidium pedatum from Bagras bayad Forskal, 1775 
and B. docmac Forskal, 1775 (Bagridae) from the Nile at Cairo. In Ethiopia, Fischthal (1973) recorded and redescribed G. pedatum from the intestine of Clarias gariepinus (as C. mossambicus). Bray and Hendrix (2007) recorded G. pedatum in Lake Malawi among Clarias gariepinus (as Clarias mosambicus). In South Africa, Matla (2012) isolated G. pedatum from the gut of Clarias gariepinus.

Characteristics of Astiotrema lazeri ElNaffar, Saoud \& Hassan, 1984 collected from the intestine of Clarias gariepinus (as Clarias lazera) from Lake Nasser at Aswan, Egypt may be a synonymy of Glossidium pedatum, according to Bray et al. (2006).

Mashego and Saayman (1989) noted that the features of Afromacroderoides lazerae Khalil, 1972, which was collected from the intestine of Clarias gariepinus (as Clarias lazera) from the White Nile near Khartoum, Sudan, were similar to those of the Plagiorchiidae, and considered $A$. lazerae a synonym of Glossidium pedatum. Dumbo et al. (2019) provided morphological and molecular studies on $G$. pedatum from Clarias gariepinus in Mozambique. They included Afromacroderoides lazerae Khalil, 1972 to be a member of Glossidium Looss, 1899 as G. lazerae. On the other hand, Afromacroderoides lazerae Khalil, 1972, has a distinct esophagus as well as a bipartite seminal vesicle. Since then, Afromacroderoides lazerae has been found in cichlids (Bray et al., 2006).

Further records of Glossidium pedatum from Egypt included those from Clarias gariepinus (Abdel-Gaber et al., 2016) or Clarias gariepinus (as C. lazera) (ElShahawi and Al-Bassel 1992, Imam et al., 1991) or from bagrid fishes (Ibraheem, 2007). This is characterized by a short prepharynx and a four lobed pharynx. The ovary and testes are both in the same position. Caeca wide ends close to the posterior testis and does not extend to the posterior extremity. Vitellaria extend from the postacetabular to the posterior testis level. As shown in Table 3, the measurements of the current specimens were comparatively consistent with those given by the previous authors.

\section{Acanthostomum spiniceps (Looss, 1896) Looss, 1899:}

Looss (1899) established the genus Acanthostomum, which included intestinal parasites of fishes and reptiles (primarily crocodiles), for the species A. spiniceps (Looss, 1896) and A. coronarium (Cobbold, 1861). In Giza, Egypt, Fischthal and Kuntz (1963a) collected $A$. spiniceps from $B$. bayad. Khalil (1963) redescribed $A$. spiniceps from $B$. bayad and $B$. docmac from the White and Blue Niles in Khartoum, Sudan. He created a new key to distinguish Acanthostomum species. Morsy et al. (2013) isolated this parasite from the intestine of Lates niloticus along the River Nile in Beni-Suef Governorate.

Further studies were provided about Acanthostomum spiniceps (Looss, 1896) from Egypt among bagrid fishes (Moravec, 1976; Tadros et al., 1978; Soliman and ElDamarany, 1995; Ibraheem, 2006; Mansour, 2012) and marine fishes (ElShahawi and Al-Bassel, 1992; Abdel-Gaber et al., 2018; Tadros et al., 2020). This species was also studied by Dollfus (1932), Pogoreltseva (1952) and Fernandez et al. (2002). This species is characterized by vitellaria extend anteriorly beyond the level of the seminal vesicle and the number of circumoral spines ranges from 23-32. Measurements of the present specimens were comparatively consistent with those provided by the previous authors as given in Table 4. The River Nile in Qena Governorate constitutes a new locality to this species.

\section{Clinostomum complantum (Rudolphi, 1814) Braum, 1899:}

Ukoli (1966a) classified $C$. complantum metacercariae based on the following characteristics: cirrus pouch on the right margin of the anterior testis in front of the ovary; genital pore on the right 
margin of the anterior testis near its middle, or occasionally displaced to its anterior margin. He also noted that the position of the genital pore is a useful character for distinguishing Clinostomum species, despite the fact that other authors have argued that this character can change with different fixation or flattening techniques (Baer, 1933), or during development (Vianna et al., 2003).

Li et al. (2018) identified Clinostomum complantum metacercariae in Myxocyprinus asiaticus from China. Maleki et al. (2018) found $C$. complantum metacercariae from some freshwater fishes in western Iran. Menconi et al. (2020) examined metacercariae of $C$. complantum in an Italian Subalpine Lake. Additional research was conducted on the metacercariae of $C$. complantum (Caffara et al, 2011; Simsek et al., 2018; Wang et al., 2017).

El-Shahawy et al. (2017) recorded metacercariae from some freshwater fishes in Qena Governorate, Egypt. They only studied the prevalence of infection and they didn't provide any morphological or morphometric description for their examined metacercaria. Measurements of the present specimens were remarkably similar with that given by Shini (2015) as shown in Table 5.

\section{Euclinostomum heterostomum (Rudolphi, 1809) Travassos, 1928:}

Euclinostomum heterostomum was reported among Tilapia spp. and Clarias sp in Egypt by Fischthal and Kuntz (1963c). Ukoli (1966b) provided a detailed morphological description of $E$. heterostomum from Tilapia zilli and $T$. heudelot. He believed that the position of the genital complex, the entrance of the uteroduct in the uterus, and the arrangement of diverticula (rather than the number of diverticula) are useful for species discrimination. He proposed the synonymy of Euclinostomum clarias, E. dollfusi, E. bhagavantami, E. charmai, E. heptacaecum, and $E$. indicum with $E$. heterostomum. Based on the mentioned criteria, he recognized $E$. heterostomum and E. multicaecum as valid species.

Kazacos and Appel (1983) recorded E. heterostomum in the Aquarium Ram, Apistogramma ramirezi (Pisces: Cichlidae) from Hong Kong. Britz et al. (1985) reported the distribution of $E$. heterostomum metacercarial infections in Oreochromis mossambicus. Euclinostomum heterostomum was isolated from the liver, spleen, and kidney of Channa punctata by Sinha et al. (1988). Saad (2007) reported metacercariae of Euclinostomum heterostomum in the kidneys of Oreochromis niloticus and Tilapia zillii in Lake Nasser, Aswan, Egypt. He did not provide morphological or morphometric descriptions for his specimens. More research was done on E. heterostomum metacercariae (Donges, 1974; Jhansilakshmibai and Madhavi, 1997; Caffara et al., 2016). As shown in Table 6, the measurements of the current specimens were remarkably similar to those provided by the previous authors.

\section{Conclusion}

In this study, six species of digenean trematodes were examined from four freshwater fish species from different regions along the River Nile in Qena and Aswan governorates, Egypt. Worms were described morphologically and morphometrically using light microscopy. More scanning, transmission electron microscopy and molecular studies are recommended to justify the exact taxonomic position of these species.

\section{Conflicts of interest}

The authors declare that there is no conflict of interest.

\section{References}

Abdel-Gaber R, Abdel-Ghaffar F, Morsy K, Bashtar AR, Saleh R (2015). New Geographical Record of Orientocreadium batrachoides (Digenea, Orientocreadiidae) of African Sharptooth Catfish Clarias 
gariepinus in Egyptian Water. International Journal of Animal Biology, 1(6): 286-291.

Attia MM, Abdelsalam M, Korany R, Mahdy OA (2021). Characterization of digenetic trematodes infecting African catfish (Clarias gariepinus) based on integrated morphological, molecular, histopathological, and immunological

examination. Parasitology Research, $1-14$.

Beverley-Burton M (1962). Some Trematodes from Clarias spp. in the Rhodesias, including Allocreadium mazoensis n. sp. and Eumasenia bangweulensis $\mathrm{n}$. $\mathrm{sp}$. and comments on the species of the genus Orientocreadium Tubangui, 1931. Proceedings of the Helminthological Society of Washington, 29(2): 103115.

Bhure DB, Nanware SS (2011). Studies on Piscian Trematode Orientocreadium batrachoides from Channa Gachua. Recent Research in Science and Technology, 3(9): 13-14.

Bhure DB, Nanware SS, Dhondge RM (2013). Studies on piscian trematode Orientocreadium batrachoides tubangui, 1931 from Channa gachua. Bulletin of Pure and Applied Sciences-Zoology, 32(2): 57-59.

Bhure DB, Nanware SS (2016). Protein contents of trematode, Orientocreadium batrachoides. Flora and Fauna, 22(2): 299-301.

Bray RA, Hendrix SS (2007). A new genus and species of macroderoididae, and other digeneans from fishes of Lake Malawi. African Journal of Parasitology, 93(4): 860-865.

Bray RA, Oosterhout C, Blais J, Cable J (2006). Astiotrema turneri n. sp. (Digenea: Plagiorchiidae) from cichlid fishes (Cichlidae:
Perciformes) of Lake Malawi, Southeastern African Zootaxa, 1319: 43-58.

Britz J, Van As JG, Saayman JE (1985). Occurrence of distribution of Clinostomum tilapiae Ukoli, 1966 and Euclinostomum heterostomum (Rudolphi, 1809) metacercarial infections of freshwater fish in Venda and Lebowa, southern Africa. Journal of Fish Biology 26: 21-28.

Caffara M, Locke SA, Cristanini C, Davidovich N, Markovich MP, Fioravanti ML (2016). A combined morphometric and molecular approach to identifying metacercariae of Euclinostomum heterostomum (Digenea: Clinostomidae). The Journal of parasitology, 102(2): 239248.

Caffara M, Locke SA, Gustinelli A, Marcogliese DJ, Fioravanti ML (2011). Morphological and molecular differentiation of Clinostomum complanatum and Clinostomum marginatum (Digenea: Clinostomidae) metacercariae and adults. Journal of Parasitology, 97(5): 884-891.

Carleton H (1967). Carleton's histological technique. 4th Ed., Oxford University Press, New Yourk, Toronto.

Dönges J (1974). The life cycle of Euclinostomum heterostomum (Rudolphi, 1809) (Trematoda: Clinostomatidae). International Journal for Parasitology, 4: 79-90

Dumbo JC, Dos Santos QM, AvenantOldewage A (2019). Morphological and molecular characterization of Glossidium pedatum Looss, 1899 and Orientocreadium batrachoides Tubangui, 1931 from sharptooth catfish, Clarias gariepinus (Burchell, 1822). African Zoology, 54(1): 43-61.

El-Mansy A (2011). Histopathology of farmed freshwater fish infested with 
different helminthes. Egyptian

Journal of Aquatic Biology and Fisheries, 15(1): 1-13.

El-Mansy A, Hamada S, Hasan S, ElSarnagawy D (2011). Histopathology of farmed freshwater fish infested with different helminthes. Egyptian Journal of Aquatic Biology and Fisheries, 15(1): 1-13.

El-Naffar MK (1970). Studies on the parasites of Nile fishes in Assiut Governorate of Egypt. Ph. D. Thesis, Fac. Sci., Assiut Univ., Assiut, Egypt.

El-Naffar MK, Hassan IM, Saoud MF (1983). A general survey of the helminth parasites of some fishes from Lake Nasser [Egypt]. Assiut Veterinary Medical Journal, 11(21): 140-184.

El-Naffar MK, Saoud MF, Hassan IM (1984). Some Trematodes from some fishes of Lake Nasser at Aswan including a new Astiotrema lazera $\mathrm{n}$. sp. Assiut Veterinary Medical Journal, 12(23): 47-60.

El-Naggar MM, Ibrahim HA, Hamada SF (1993a). Redescription of Orientocreadium batrachoides Tubangui, 1931, a digenean intestinal parasite of Clarias lazera in Egypt. Journal of Egyptian German Society of Zoology, Invertebrate Zoology and Parasitology, 10: 157-170.

El-Naggar MM, Ibrahim HA, Hamada SF (1993b). Scanning and transmission electron microscope observations on the general body tegument and ventral pads of the digenean Orientocreadium batrachoides Tubangui, 1931. Journal of the Egyptian-German Society of Zoology, Invertebrate zoology and parasitology, 10: 245-264.

El-Seify MA, El-Dakhly KM, Metwally A, Mohammed ES, Fawaz MM (2017). Prevalence of gastrointestinal
SVU-IJVS, 4 (4): 68-84

parasites infecting fish (Clarias gariepinus) in Qena governorate. Kafrelsheikh Veterinary Medical Journal, 15(1): 65-88.

El-Shahawi GAZ, Al-Bassel DA (1992). A general survey of the helminth parasites infecting common fishes in some inland water in Egypt. Proceedings of the Zoological Society AR Egypt, 23: 227-241.

El-Shahawy IS, El-Seify MO, Metwally AM, Fwaz MM (2017). Survey on endoparasitic fauna of some commercially important fishes of the River Nile, southern of Egypt (Egypt). Revue De Medecine Veterinaire, 168(4-6): 126-34.

El-Siefy AMHM (2008). Parasitological and biological studies on some parasites of River Nile Fishes in ElMinia Governorate. M. Sc. Thesis, Fac. Sci. El-Minia Univ., Egypt.

Fernandes BMM, Pinto RM, Cohen SC (2002). Report on two species of Digenea from marine fishes in Brazil. Brazilian Journal of Biology, 62(3): 459-462.

Fischthal JH, Kuntz RE (1963a). Trematode parasites of fishes from Egypt. Part V. Annotated record of some previously described forms. Journal of Parasitology, 49(1): 91- 98.

Fischthal JH, Kuntz RE (1963b). Trematode Parasites of Fish from Egypt. Part VII Orientocreadium batrachoides Tubangui 1931 (Plagiorchioidea) from Clarias lazera with a review of the genus and related forms. Journal of Parasitology, 49(3): 451-464.

Fischthal JH, Kuntz RE (1963c). Trematode parasites of fishes from Egypt. Part VI. The metacercaria of Euclinostomum heterostomum (Rudolphi, 1809) Travassos, 1928 (Clinostomidae). with a review of the 
genus. Transaction of the American Microscopical Society, 82: 335-342.

Hamada SF (2002). Scanning electron microscope observation on the surface features of the digenean Astiotrema impletum (Looss, 1899) Looss, 1900. Journal of the Egyptian Society of Parasitology, 32(2): 487500 .

Hassan M (2006). Parasites of native and exotic freshwater fishes in the southwest of Western Australia. Ph.D. Thesis, Murdoch University.

Ibraheem MH (2007). Surface ultrastructure of the plagiorchid trematode Glossidium pedatum Looss 1899 from bagrid fish in Egypt. Acta Zoologica, 88(2): 173-178.

Jhansilakshmibai K, Madhavi R (1997). Euclinostomum heterostomum (Rudolphi, 1809) (Trematoda): Lifecycle, growth and development of the metacercaria and adult. Systematic Parasitology, 38: 51-64.

Karar YF, Blend CK, Dronen NO, Adel A (2021). Towards resolving the problematic status of the digenean genus Astiotrema Looss, 1900: An updated concept and revision of species composition for Astiotrema (sensu stricto). Zootaxa, 4991(1): 3672 .

Kazacos KR, Appel GO (1983). Euclinostomum heterostomum metacercarie (Trematoda: Clinostomatidae) from the Aquarium Ram, Apistogramma ramirezi (Pisces: Cichlidae). Proceedings of Helminthological Society, 50(1): 103107.

Khalil LF (1969). Studies on the helminth parasites of freshwater fishes of the Sudan. Journal of Zoology, 158: 143170.

Li F, Liu XH, Ge HL, Xie CY, Cai RY, Hu ZC, Zhang YG, Wang ZJ (2018). The discovery of Clinostomum complanatum metacercariae in farmed Chinese sucker, Myxocyprinus asiaticus. Aquaculture, 495: 273-280.

Looss A (1896). Recherches sur la faune parasitairo de 1' Egypte. Premiere partie. Mem. Inst. Egypte, 3: 1-252.

Looss A (1899). Weitere Beiträge zur Kenntnis der Trematoden-Fauna Aegyptens, zugleich Versuch einer naturlichen Gliedering des Genus Distomum Retzius. Zoologische Jahrbucher, Abteilung für Systematik, 12: 521-784.

Looss A (1900). Nachträgliche Bemerungen $\mathrm{Zu}$ den Namen der von Mir vergeschlagenen Distomum gattungen. Zoologischer Anzeiger, 23: 601-608.

Mahmoud N, Abu El-Wafa S, El-Aal A (1997). Studies on some enteric helminthes of Nile fishes from Sharkia province, Lower Egypt. Egyptian Journal of Aquatic Biology and Fisheries, 1(2): 431-451.

Maleki L, Heidari H, Ghaderi E, Rostamzadeh J (2018). Occurrence and description of Clinostomum complanatum (Rudolphi, 1819) metacercariae in freshwater fishes from Gheshlagh basin, West of Iran. Iranian Journal of Animal Biosystematics, 14(2): 91-103.

Mansour MF (2012). Ultrastructure of the spermatozoon of Acanthostomum spiniceps

(Digenea: Acanthostomidae), a parasite of Bagrus spp. (Siluriformes: Bagridae). Parasitology Research, 110(4): 13571362.

Mashego SN (1977). A seasonal investigation of the ecto- and endoparasites of the barbel, Clarias gariepinus (Burchell, 1822) in Lebowa, South Africa. M. Sc. 
dissertation, University of the North, South Africa.

Menconi V, Manfrin C, Pastorino P, Mugetti D, Cortinovis L, Pizzul E, Pallavicini A, Prearo M (2020). First Report of Clinostomum complanatum (Trematoda: Digenea) in European Perch (Perca fluviatilis) from an Italian Subalpine Lake: A Risk for Public Health? International journal of environmental research and public health, 17(4), 1389

Mohamed IB (1996). Pathological changes in some fish eating mammals as a result of consumption of fish infested with encysted metacercariae. Ph. D. Thesis, (Pathology), Fac. Vet. Med. Cairo University.

Moravec F (1976). On two Acanthostomatid trematodes, Acanthostomum spiniceps (Looss, 1896) and A. absconditum (Looss, 1901), from African bragrid fishes. Folia Parasitology, 23(3): 201-206.

Moravec F (1977). Some digenetic trematodes from Egyptian freshwater fishes. Věstník Československé společnosti zoologické, 41: 52-67.

Moravec F, Sey O (1989). Some trematodes of freshwater fishes from North Vietnam with a list of recorded endohelminths by fish hosts. Parasitology, 36(3): 243-262.

Morsy K, El-Fayoumi H, Ali S (2013). Acanthostomum spiniceps (Digenea: Cryptogonimidae: Acanthostominae), a parasite of the African snook Lates niloticus (Perciformes: Latidae). A light and scanning electron microscopic study. Journal of the Egyptian Society of Parasitology, 43(3): $1-8$.

Odhner T (1911). Nordostafrikanische Trematoden, grösstenteils vom Weissen Nil., I. Fascioliden. Result of the Swedish Zoological Expedition to
Egypt and the White Nile, 1901, 23: 1-170.

Paperna I, Dzikowski R (2006). Digenea (Phylum Platyhelminthes): In fish diseases and disorders, Protozoan and metazoan infections, Vol. 1. (Ed: Woo, P.T.K.). CAB International, Wallingford, UK, pp: 345-390.

Rai P (1970). On the clinostomatid metacercaria in come of our edible fishes and remarks on the pathological significance. Indian Journal of Animal Sciences, 40(2): 189-198.

Ruangpan S (1989). Life cycle of Orientocreadium batrachoides Tubangui, 1931 (Trematoda: Allocreadiidae) in Clarias macrocephalus Gunther, 1864. Thai Fisheries Gazette, 42: 436-441.

Saad AI (2007): Acomparative study of different metacercarial infections in two freshwater fishes from Lake Nasser, Nile River and hatcheries at Aswan-Egypt. Journal-Egyptian German Society of Zoology, 51(D): 107-125.

Simsek E, Yildirim A, Yilmaz E, Inci A, Duzlu O, Onder Z, Ciloglu A, Yetismis Y, Pekmezci GZ (2018). Occurrence and molecular characterization of Clinostomum complanatum (Trematoda: Clinostomidae) in freshwater fishes caught from Turkey. Parasitology research, 117(7): 2117-2124.

Sinha AK, Sinha C, Nikhil R (1988). Studies on yellow grub disease of freshwater fish Channa punctatus (Bloch). Current Science, 57: 218219.

Sirikantayakul S (1976). Observations on the life cycle and egg shell of Orientocreadium batrachoides Tubangui, 1931 (Trematoda: Allocreadiidae) in Clarias 
macrocephala Gunther 1864. Ph.D. Thesis, University of the Philippines.

Soliman FM, El-Damarany M (1995). Prevalence of helminth infection among some Nile fishes in relation to some biological aspects. Journal of the Egyptian German Society of Zoology, 16: 253-275.

Tadros G, Iskandar AR, Wassef NA (1978). On some intestinal trematodes from the Nile and Red Sea fishes with a histopathological study of their habitat Journal of the Egyptian Society of Parasitology, 8: 383-392.

Tang CC, Lin SM (1973). On the lifehistory of Orientocreadium batrachoides Tubangui, 1931 with a consideration on the phylogeny of the superfamily Plagiorchioidea. Acta Zoologica Sinica, 19: 11-25.

Tepe Y, Oguz MC, Belk M, Özgen R (2013).

Orientocreadium

batrachoides Tubangui, 1931

(Orientocreadiidae): The only

Trematode Parasite of Clarias

gariepinus (Burchell, 1822)

(Clariidae) from the Asi River

(Southern Turkey). Türkiye

Parazitolojii Dergisi, 37(3): 203- 207.

Ukoli FMA (1966a). On Clinostomum tilapiae n. sp., and $C$. phalacrocoracis Dubois, 1931 from Ghana, and a discussion of the systematics of the genus Clinostomum Leidy, $1856 . \quad$ Journal of Helminthology, 40: 187-214.

Ukoli FMA (1966b). On Euclinostomum heterostomum (Rudolphi, 1809). Journal of Helminthology, 40: 227234.

Vankara AP, Mani G, Vijayalakshmi C (2011). A report on various digenetic metacercariae from the freshwater fishes of River Godavari, Rajahmundry. Journal of Parasitic Diseases, 35(2): 177-185. van Rensburg, JC, van As JG, King PH (2013). New records of digenean parasites of Clarias gariepinus (Pisces: Clariidae) from the Okavango Delta, Botswana, with description of Thaparotrema botswanensis sp. n. (Plathelminthes: Trematoda). African Invertebrates, 54(2): 431-446.

Vianna RT, Pereira J, Brandao DA (2003). Ontogenetic variation of metacercariae of Clinostomum complanatum (Rudolphi, 1814) (Digenea: Clinostomidae). Comunicacoes do Museu de Ciencias e Tecnologia da PUCRS. Serie Zoologia, Porto Alegre, 16: 223-243.

Wang ML, Chen HY, Shih HH (2017). Occurrence and distribution of yellow grub trematodes (Clinostomum complanatum) infection in Taiwan. Parasitology research, 116(6): 1761-1771.

Yamaguti S (1971). Synopsis of digenetic trematodes of vertebrates Tokyo. Vols I and II, 1074 pp.

Yeh LS, Fotedar DN (1958). A review of the trematode genus Astiotrema in the family Plagiorchidea. Journal of Helminthology, 32: 17-32.

Zhokhov AE, Morozova DA, Pugacheva MN (2017). Trematodes (Plathelminthes) of Clarias gariepinus (Pisces: Clariidae) in Lake Tana, Ethiopia. Inland Water Biology, 10(2): 219-227. 